Published in final edited form as:
J Radiol Nurs. 2014 December ; 33(4): 166-180. doi:10.1016/j.jradnu.2014.08.004.
Pediatric Sedation: Using Secondary Data to Describe
Registered Nurse Practice in Radiology
Nancy Crego, PhD, RN, CCRN
Georgetown University
Abstract
Children, often require sedation for procedures due to their developmental level and difficulty
complying with positioning. There are few studies that describe nurse sedation practices or
adverse events. Studies of pediatric sedation care have small sample sizes that are inadequate to
detect adverse events. This study reports practices and outcomes of sedation delivered to children
from infancy up to 14 years of age, that were monitored only by registered nurses (RNs) during
diagnostic radiology procedures drawn from a sample of 12,584 cases from the Pediatric Sedation
Research Consortium (PSRC) database. There were 727 adverse events (5.78\%). However, no
deaths, cardiac arrests, intubations or aspirations were reported in this sample. The most common
adverse event was inadequate sedation/agitation/delirium 196 (155.8/10,000) and desaturation
below baseline for greater than 30 seconds 173 (138/10,000). Further research comparing sedation
practices and outcomes by type of providers, including nurses, are necessary to improve practice.

\title{
Keywords
}

sedation; pediatrics; nurse sedation; radiology nursing; adverse events; pediatric sedation research consortium

\begin{abstract}
The growing demand for diagnostic procedures such as Magnetic Resonance Imaging (MRI) and Computerized Tomography (CT) scans for children of all ages has led to an increased demand for procedural sedation services (Havidich \& Cravero, 2012). Procedural sedation is "a technique of administering sedative or dissociative agents with or without analgesics to induce a state that allows the patient to tolerate unpleasant procedures while maintaining cardiorespiratory function. Procedural sedation and analgesia (PSA) is intended to result in a depressed level of consciousness that allows the patient to maintain oxygenation and airway control independently" (Godwin et al., 2005, p. 178). In order to meet the need for sedation services in this population, numerous specialties such as radiologists and registered nurses (RNs) with varying education and experience provide sedation (Havidich, \& Cravero, 2012).
\end{abstract}

(C) 2014 American Radiological Nurses Association. Published by Mosby, Inc. All rights reserved.

Corresponding Author: Nancy Crego, 1201 N Garfield Street \#507, Arlington, Va. 22201.

Presented At American Association of Colleges of Nurses Doctoral Education Conference, Naples, Fl. January 31, 2014

Publisher's Disclaimer: This is a PDF file of an unedited manuscript that has been accepted for publication. As a service to our customers we are providing this early version of the manuscript. The manuscript will undergo copyediting, typesetting, and review of the resulting proof before it is published in its final citable form. Please note that during the production process errors may be discovered which could affect the content, and all legal disclaimers that apply to the journal pertain. 


\section{Sedation In Pediatrics}

The goals of sedation in children are to maintain safety, minimize discomfort, decrease anxiety, minimize psychological trauma, increase cooperation with exam requirements such as immobilization in order to complete the procedure, and to return the child to their preprocedural physical and cognitive state (Coté \& Wilson, 2006). Children under six years of age often require deep levels of sedation in order to gain the cooperation necessary to complete diagnostic procedures (Coté \& Wilson, 2006). Sedated children are at risk for serious adverse events such as apnea, airway obstruction and hypotension (Coté \& Wilson, 2006). Adverse responses to sedation cannot be eliminated, but can be mitigated by assuring that appropriate screening, medications, equipment, monitoring and personnel are in place to provide sedation (Coté \& Wilson, 2006).

\section{Sedation Outside the Operating Room}

Until the 1980s, the practice of sedation was completed in the operating room by anesthesiologists (Krauss \& Green, 2008). In the last 20 years, the demand for sedation outside the operating room, in locations such as radiology, has led to the expansion of sedation services by many non-anesthesia provider specialists such as staff RNs, advanced practice nurses, and physician specialists such as intensivists and radiologists (Krauss \& Green, 2008). This study focused only on staff RN sedation providers; advanced practice nurses such as nurse practitioners and Certified Registered Nurse Anesthetists (CRNAs) were excluded. Although RNs often provide sedation care directly to patients, institutional oversight of sedation generally remains with physician specialists, such as anesthesiologists, who provide expertise in the development of sedation protocols and assuring sedation quality (Krauss \& Green, 2008). The current model of varying institution level sedation practice and administrative responsibility means that sedation systems differ depending on location. This inherent variation in sedation care has made research difficult. Many studies about sedation analyze practices and outcomes of physician sedation providers but research on RN sedation providers is limited (Couloures, Beach, Cravero, Monroe, \& Hertzog, 2011).

Studies of RN pediatric sedation care are similarly limited and involve small sample sizes, describe implementation of RN sedation services, only include data on one location, or compare sedative medication regimes (Beebe et al., 2000; Blumke \& Breiter, 2000; Gozal \& Gozal, 2008; Lavoie, Vezina, Paul-Savoie, Cyr, \& Lafrenaye, 2012; Shah et al., 2011; Srinivasan, Turmelle, DePalma, Mao, \& Carlson, 2012; Sury, Hach, Deeley, Dicks-Mireaux, \& Chong 1999; Sterni, Beck, Cole, Carlson, \& Turmelle, 2008; Woodthorpe, Trigg, Gurney, $\&$ Surry, 2007). This study was undertaken to describe practices and outcomes of pediatric sedation by RNs in radiology to determine the patient characteristics, medications delivered, monitoring practices and outcomes of sedation by RNs in children during diagnostic MRI, CT scan, and ultrasound procedures.

\section{Pediatric Sedation Guidelines}

In 2006, the American Academy of Pediatrics (AAP) updated guidelines for the monitoring and management of pediatric patients during and after procedural sedation (Coté \& Wilson, 2006). The updated document was modified to incorporate similar language, definitions of 
sedation and monitoring guidelines found in sedation regulations such as the Joint Commission sedation standards (Joint Commission International, 2011). The AAP does not include any information regarding the $\mathrm{RN}$ role in providing or assisting with sedation or data regarding RN sedation practice (Coté \& Wilson, 2006). The AAP guidelines also utilize the American Society of Anesthesiologists (ASA) (American Society of Anesthesiologists, 2002) guidance document for Sedation and Analgesia by Non-Anesthesiologists in describing safe pediatric sedation practice.

\section{American Society of Anesthesiologists}

The ASA has published several advisories, statements, and guidelines concerning sedation by non-anesthesiologists, which have set the standard for sedation care in the United States. There are four levels of sedation described by the ASA, ranging from minimal to general anesthesia, occurring on a continuum (see Table 1). In the pediatric population a deep level of sedation is often required in order to complete diagnostic procedures (Coté \& Wilson, 2006; Gozal \& Gozal, 2008). The ASA also established standards for the care of the sedated patient and an anesthesia risk score called the ASA score (see Table 2). Several studies on pediatric sedation have demonstrated that children, especially those under age six, are at risk of unintentionally moving from the intended level of sedation to a deeper than intended level of sedation (Coté \& Wilson, 2006). Therefore, sedation providers are required to have the skills necessary to rescue patients from a deep level of sedation during the procedure (Coté \& Wilson, 2006).

The most recent ASA statements on granting privileges for moderate sedation defines nonanesthesiologist sedation practitioners as "licensed physicians, dentists, or podiatrists who have not completed postgraduate training in anesthesiology but are specifically trained to personally administer or supervise the administration of moderate sedation" and supervised sedation practitioners as "a licensed registered nurse, advanced practice nurse or physician's assistant who is trained to administer medications and monitor patients during moderate sedation under the direct supervision of a nonanesthesiologist sedation practitioner or an anesthesiologist" (ASA, 2010, p. 3). In the latest ASA advisory statement on granting privileges for deep sedation, only non-anesthesiologist sedation practitioners and anesthesia professionals such as anesthesiologists and CRNAs are qualified to administer deep sedation (American Society of Anesthesiologists, 2010). The ASA advisory statements do not provide any information on current RN sedation practice, whether or not RNs are providing sedation care under direct supervision, the level of sedation, types of medications RNs deliver or the outcomes of $\mathrm{RN}$ sedation.

\section{Sedation in Radiology}

Children often receive sedation for procedures in radiology such as CT scans and MRI, which may require moderate to deep levels of sedation (Coté \& Wilson, 2006). The radiology environment presents many challenges to sedation providers such as difficulty in visualizing the patient during the procedure because the patient is placed inside an enclosed area and must remain still (American Society of Anesthesiologist, 2009). Assessing level of responsiveness would also interfere with successful completion of the exam for which the patient was sedated (Coté \& Wilson, 2006). Monitoring pulse and respiratory rate may be 
impeded during MRI procedures because of the incompatibility of equipment such as standard electrocardiogram machinery and leads in the presence of static and dynamic magnetic fields and radiofrequency energy emissions that pose safety hazards to the patient (American Society of Anesthesiologists, 2009). The radiology environment presents several challenges to RN sedation providers. Prior studies have provided data from single locations, using sample sizes that are underpowered to detect adverse events. Samples in the tens of thousands are required to estimate adverse sedation events (Cravero et al., 2006).

\section{RN Sedation Outcomes in Radiology}

Bluemke and Breiter (2000) measured the effect of RNs on safety and effectiveness on sedation for MRI. The study sample of 4,761 sedated patients was obtained from a sedation database at Johns Hopkins Hospital from an eight-year period of data collection (Bluemke \& Breiter, 2000). RNs followed a sedation protocol that included a pre-sedation history and review of systems conducted by a radiologist (Bluemke \& Breiter, 2000). Patients were eligible for RN sedation if they were ASA I or II and met fasting guidelines (Bluemke \& Breiter, 2000). Patients received sedative medications based on age and weight to induce conscious/moderate to deep sedation with monitoring during the procedure and recovery completed by the sedation RN with physician oversight (Bluemke \& Breiter, 2000).

There were only $20(0.42 \%)$ complications observed and no deaths reported (Bluemke \& Breiter, 2000). Sedation failure rates were reported for each medication on the sedation protocol and ranged from a low of $4.8 \%$ for chloral hydrate to $13.1 \%$ for oral diazepam (Bluemke \& Breiter, 2000). In this study the mean time to onset of sedation and the variability in the time to onset of sedation were measured to determine the predictability of sedation (Bluemke \& Breiter, 2000). Comparisons of sedation times were made between a group of four sedation radiology RNs, radiology RNs who did not routinely perform sedation, and inpatient floor nurses who rarely provided sedation in radiology (Bluemke \& Breiter, 2000). The group of four sedation radiology RNs had significantly shorter mean time to onset of sedation and demonstrated the least variability in sedation times when compared to the other RNs in the study (Bluemke \& Breiter, 2000).

Lavoie et al., (2012) described outcomes of a pediatric intensivist supervised RN led sedation program for painful and painless procedures using a retrospective review of records for a one-year period. Sedation RNs followed stringent patient selection criteria including: "ASA that had to be less than 3, no active upper respiratory tract infection, no acute neurologic condition, no high fever, and no snoring or sleep apnea" (Lavoie et al., $2012 \mathrm{p}$. 2). The RNs in this sedation program were expected to provide sedation and monitor patients during many types of procedures such as imaging, endoscopy, and bone marrow aspiration (Lavoie et al., 2012). In this study 555 procedures were performed on 448 children ranging in age from 3 weeks to 18 years; MRI was the most frequent (24\%) followed by CT scan (10\%) (Lavoie et al., 2012).

There were no deaths, aspirations, unexpected admissions or resuscitations reported. There was a $22 \%$ complication rate with $8 \%$ of these consisting of oxygen saturation less than $90 \%$, bradycardia or hypotension more than 2 standard deviations below the normal for the age of the child, and a 5\% rate of sedation failure due to patient agitation, inadequate 
sedation and failure to perform the procedure (Lavoie et al., 2012). The authors found that outcomes of this nurse led sedation program were similar to other programs reported in the literature (Lavoie et al., 2012).

\section{RN Sedation Training}

Reports of RN led sedation services provide general descriptions of "sedationist" responsibilities such as screening patients using predetermined criteria and monitoring the patient during the procedure (Sterni et al., 2008; Woodthorpe et al., 2007). The qualifications and training of a nurse sedationist varies, as there are no national certifications or qualifications that have been developed for RNs in this role. Woodthorpe et al., (2007) describe the use of tutorials by a pediatric anesthesiologist along with training including principles and hazards of sedation, identification of common sedation contraindications, and a preference for RNs with experience in the intensive care unit, emergency department, or post-operative recovery. The RNs in this sedation service also demonstrated airway management skills on anesthetized children in the operating room with reassessment of skills every three months (Woodthorpe et al., 2007).

There were 926 children scheduled for MRI with sedation: 5.5\% were denied sedation due to various contraindications and 780 required sedation with a 95\% success rate (Woodthorpe et al., 2007). Seven children experienced incidents described as "clinically significant" but none required admission to the hospital and four of them successfully completed the procedure (Woodthorpe et al., 2007). The study authors did not describe if RNs performed airway or other interventions during sedation, however they did opine that the skill and judgment of the RN to refer children that would not be effectively sedated or were at risk for complications was a factor in making this RN sedation service successful and safe (Woodthorpe et al., 2007).

Sterni et al., (2008), described a sedation team model overseen by anesthesiologists in which the sedation qualified RN completed the same training as sedation qualified non-anesthesia physicians. All sedation providers completed a 60 to 80 hour lecture based anesthesia airway management course and also completed mentored skills training; credentialing was approved at the discretion of the chief of anesthesia (Sterni et al., 2008). RN sedation providers were limited to using only oral or rectal chloral hydrate or midazolam and could administer pentobarbital for sedation via the intravenous or intramuscular route (Sterni et al., 2008). The RN sedation protocol limited RNs to sedate children with an ASA I score and no history of sedation or problems related to sedation (Sterni et al., 2008). There were no outcome data reported on the sedation team.

\section{Limitations of RN Sedation Studies}

Several studies have reported RN sedation outcomes such as adverse events, but the sample sizes for most of these studies are inadequate to detect mortality and morbidity associated with sedation (Cravero et al., 2006). Multi-center data on sedation and large sample sizes are necessary in order to estimate the incidence of mortality and other rare sedation events (Cravero et al., 2006). Recently, studies completed by the Pediatric Sedation Research Consortium (PSRC), have used data from their large multi-center database to describe many 
aspects of pediatric sedation by non-anesthesia providers. A study using a total of 30,037 records from the PSRC found that 1,601 recorded some type of complication, which is a 5.3\% incidence rate (Cravero et al., 2006). Complications included one incident of aspiration and one cardiopulmonary resuscitation; both ended with discharge of the patient in good condition after brief hospitalization (Cravero et al., 2006).

Although the PSRC collects data on sedation by many types of non-anesthesia providers including RNs, the results that have been reported for RNs have been aggregated with other providers. For example in a study by Couloures et al., (2011) complication rates depending on provider specialty were described but RN provider data were included in the "other" category that also included radiologists, dentists, surgeons, and pediatric resident/fellows, among others (Couloures et al., 2011). The present study will provide a description of practices by RNs that deliver and monitor sedation to children for diagnostic MRI, CT scan, and ultrasound, performed in the radiology department using data from the PSRC database.

\section{Research Design and Methods}

\section{Research Question}

What are the patient characteristics, medications delivered, monitoring practices and outcomes of sedation by RNs in children during diagnostic MRI, CT scan, and ultrasound procedures?

\section{Methods}

A descriptive research design using secondary data from the PSRC database was used to examine patient characteristics and sedation practices and outcomes by RNs on children in diagnostic radiology. Descriptive data on physician sedation providers has been previously reported using the PSRC database (Cravero et al., 2006). These data are presented to provide a means for comparison. The PSRC has reported on several aspects of sedation care including adverse event rates, outcomes associated with sedative medications, and physiologic monitoring practices during procedural sedation (Couloures et al., 2011; Cravero et al., 2006; Cravero, Beach, Bilke, Gallagher, Hertzog, 2009; Langhan, Mallory, Hertzog, Lowerie, Cravero, 2012). Data collected prospectively by member institutions is submitted to the database using a web based data collection tool (Cravero et al., 2006).

\section{Data Collection}

The PSRC consists of 35 member institutions that use a standard method of collecting pediatric sedation data and assures the integrity of data collection procedures through individual member institution primary investigators (Cravero et al., 2006). Sites are located in the Northeast, South, Mid-West and Western United States and there are sites in Israel, Canada and the Netherlands (Cravero et al., 2006). The institutions include children's hospitals, community hospitals and academic centers. Participating institutions obtain institutional review board approval to prospectively collect data on pediatric sedation and designate a primary investigator (Cravero et al., 2006). Each PSRC site selects a location of their choice within their institution to collect data via a web based data collection tool (Cravero et al., 2006). Data are entered by member institutions via a Sybase database 
management system that presents one question per screen; subsequent questions are generated based on prior responses and are entered on standard answer sets using a series of check boxes with the ability to add free text if necessary (Cravero et al., 2006). The software validates the input as it is entered in order to decrease errors (Cravero et al., 2006). The study web site and data entry portal are secure and require authentication in order to input data (Cravero et al., 2006). All participating institutions and primary investigators are blinded to data submitted by other individual institutions (Cravero et al., 2006). Study authors are blinded to individual institution data in order to decrease the possibility that member institutions would withhold adverse event data due to concerns related to their standing in the consortium or the institution's reputation (Cravero et al., 2006).

Primary investigators are required to perform periodic inventories of data submissions. More than $90 \%$ of cases must be reported and primary investigators must submit a separate count of sedations performed in the study location and compare it to the actual cases submitted to the PSRC database. This assures that data are not selectively submitted. Audits are performed on $5 \%$ of the submitted cases to ensure data integrity (Couloures et al., 2011). Each site selects the locations within their institution that contribute to the database.

\section{Protection of Human Subjects}

This study was submitted to the University of Virginia institutional review board and was exempt under Health and Human Services regulation 4 (45 CFR 46.101(b)(4)). PSRC data are de-identified to meet the Health Insurance Portability and Accountability Act and encrypted when transferred from the member institution to assure data security (Cravero et al., 2006).

\section{Data Management}

An encrypted data file was obtained from the PSRC consisting of one record for each case meeting the study inclusion criteria as listed in Table 3 . The data file was downloaded into a password-protected directory on a University of Virginia UNIX system. Data were examined, checked, and cleaned of errors. A data set for analysis was prepared using Statistical Analysis System (SAS, Inc., Cary, NC, USA), to translate data into a file for analysis. Data file cases and variables were screened and evaluated for patterns of missing data. There were two variables added to the PSRC web based data collection tool after data collection started. These two variables had greater than 5\% missing data, but these variables were not used in this study so the cases were retained in the study sample.

\section{Sample}

A detailed description of the study sample size and selection criteria is provided Table 3 . Inclusion criteria were: children up to and including the age of 14 years and sedated for diagnostic MRI, CT scan, or ultrasound from January 2005 to September 2007. Cases in which only RNs monitored patients and delivered medications were included. Study exclusion criteria were children over the age of 14 years and 1 month; children that were either monitored or received medications by an RN and any other sedation provider; cases in which the ASA score was missing; and cases in which interventional radiologic studies were 
completed and children receiving local anesthetics. The final study sample size was 12,584 cases.

\section{Data Elements}

The data elements collected by the PSRC were established by the consortium using AAP, ASA and American College of Emergency Physician guidelines that have been previously outlined; a review of the literature and the consensus of the consortium membership (Cravero et al., 2006). Data collection elements consist of three major categories: 1) sample characteristics, 2) role of sedation provider, and 3) procedure characteristics. Examples of the variables associated with each of these are listed below and further details are provided on Tables 4 and 5. An exhaustive list of the PSRC study variables is available at http:// an.hitchcock.org/PediatricSedationRC/.

Sample characteristics-The data collected for this study included age, weight, sex, ASA status, category of primary diagnosis (that is, the type of primary indication for the procedure being performed) and category of coexisting diagnosis (see Table 4) (Cravero et al., 2006). There are 24 possible primary and 24 possible coexisting diagnosis categories that can be selected on the web based data collection tool. The majority of the study sample $\mathrm{n}=6,253$ cases $(49.69 \%)$ were under 25 months of age. Most sample cases had a primary or coexisting neurological diagnosis category $n=9,235(73.38 \%)$ of the sample.

Sedation provider information-Data collected on cases with RNs delivering and monitoring sedation are listed in Table 5 and includes the provider responsible for oversight of the sedation. The provider responsible for oversight of sedation category only includes providers responsible for the sedation case but does not mean the provider had contact with the patient (Cravero et al., 2006). The role of sedation providers is not mutually exclusive. For example it is possible for different providers to monitor the same sedation case. However, this study sample $(\mathrm{n}=12,584)$ only included cases in which an $\mathrm{RN}$ was the only provider delivering and monitoring sedation.

Diagnostic radiology procedure characteristics—Data collected about the procedure includes the procedure performed, medications and monitoring equipment used, planned and actual depth of sedation achieved, and complications (see Table 5) (Cravero et al., 2006). MRI scans were the most common type of diagnostic radiology procedure $\mathrm{n}=8,802(70.06 \%)$ in the sample and $\mathrm{n}=12,663(100.79 \%)$ cases had MRI or CT scans. In some cases more than 1 procedure was completed on a case, such as an MRI and CT scan.

\section{Results}

The sample of 41,392 radiology procedures for MRI, CT scan and ultrasound had $n=12,584$ (30.42\%) cases where patients received medications and were monitored only by RNs during sedation (see Table 3). Deep sedation was planned for $n=1,741$ (13.84\%) of the cases and achieved in $\mathrm{n}=1,723$ (13.69\%) (see Table 5). Moderate sedation was achieved by RNs in $\mathrm{n}=10,422$ cases $(82.82 \%)$. ASA scores, which represent anesthesia risk, were compared to a sample of 30,037 sedation cases from the PSRC database, reported by Cravero et.al., (2006) in which physicians, advance practice registered nurses, including pediatric nurse 
practitioners and physician assistants were the providers responsible for sedation. ASA scores for the RN alone sample were ASA I $n=4,764$ (37.86\%) and ASA II $n=6,310$ (50.14\%); the percentage of the RN alone sample ASA scores were similar to the physician provider group presented by Cravero et al., (2006). ASA scores for the RN alone sample used for this study and the PSRC sample of physicians and advanced practice nurses reported by Cravero et al., (2006) are presented in Figure 1. The ASA III scores for the RN group were $n=1,374(10.9 \%)$ and $n=3,479(11.60 \%)$ for the physician group (Cravero et al., 2006). The similarity in ASA III scores is notable, as sedation guidelines recommend anesthesia or other subspecialty consultation due to increasing sedation risk for these patients (Coté \& Wilson, 2006).

\section{Sedation Monitoring Equipment Use}

Monitors used by RN providers during diagnostic radiology procedures are presented in Figure 2. Langhan et al. (2012) reported monitor use in 17,033 radiology cases and 50,094 MRI cases; the results from both samples are presented on Figure 2 for comparison. Langhan et al., (2012) reported electrocardiogram use of $n=34,089(67 \%)$ for MRI, $n=$ $9,364(55 \%)$ for radiology procedures but it was $\mathrm{n}=1,848(15 \%)$ in the $\mathrm{RN}$ provider sample. $\mathrm{RN}$ sedation providers also used capnography (ETCo2) monitoring in $\mathrm{n}=3,325(26 \%)$ of cases, while the MRI sample reported by Langhan et al., (2012) used ETCo2 monitoring in MRI for $\mathrm{n}=38,964(77 \%)$ of cases. Blood pressure monitoring for the MRI sample reported by Langhan et al., (2012) was $n=43,062(85 \%)$ for MRI and $n=13,315(78 \%)$ for all radiology cases but was $\mathrm{n}=5,556(44 \%)$ for $\mathrm{RN}$ sedation providers.

\section{Adverse Events}

In the RN sample there were no deaths, cardiac arrests, intubations or aspirations reported. Cases with only RN sedation providers had $\mathrm{n}=727$ (5.78\%) adverse events with the adverse event category respiratory complications being the most common $n=297(2.36 \%)$ (see Table 5). However, the lack of published descriptive statistical data on RN sedation adverse events in a large sample of pediatric patients makes it problematic to evaluate the results of this study. Thus, comparison data on adverse events from a sample of 30,037 children published by Cravero et al., (2006) using PSRC data are reported per 10,000 cases in Figures 3 and 4.

The data from Cravero et al., (2006) included physician and advance practice sedation providers; patients underwent 12 categories of procedures including radiologic. When adverse events were examined in the $\mathrm{RN}$ alone provider sample, the most common type of adverse event requiring intervention was prolonged sedation with a rate of $\mathrm{n}=53$ $(42.1 / 10,000)$ Cravero et al. (2006) reported $n=41(13.6 / 10,000)$ rate of prolonged sedation (see Figure 3). In the Cravero et al., (2006) physician provider sample, the most common adverse event type was incomplete procedure, with an $n=267(88 / 10,000)$ rate while in the $\mathrm{RN}$ alone sample the rate was $\mathrm{n}=1(0.7 / 10,000)$ (see Figure 3$)$. However, the most common adverse event type in the $\mathrm{RN}$ alone sample was inadequate sedation/agitation/delirium $\mathrm{n}=$ 196 (155.8/10,000) (see Figure 3); this adverse event type was not reported by Cravero et al., (2006). Adverse event types that may not require intervention such as desaturation (defined as an oxygen saturation below $90 \%$ for greater than 30 seconds) was the most 
common type of adverse event in the Cravero et al., (2006) sample $n=470(156.5 / 10,000)$ and was $n=173(138 / 10,000)$ in the RN only sample. Vomiting in a non-gastrointestinal procedure was more common in the RN sample $\mathrm{n}=108(86 / 10,000)$ than in the comparison group $n=142(47.2 / 10,000)$ (see Figure 4). Vomiting was the second most common type of adverse event that may not require intervention in both the RN only and Cravero et al., (2006) samples (see Figure 4).

\section{Medication Use}

In the present study, $\mathrm{n}=1,723(13.69 \%)$ of the sample was deeply sedated. RNs administered only one medication 6,885 times $(54.71 \%)$ of the sample (see Table 5). Medication use by RNs (see Figure 5) illustrates the wide variety of medications used for sedation. Fentanyl, an opioid analgesic, was administered in 9.2\% $(n=1,158)$ of $\mathrm{RN}$ sedation cases in comparison to $8 \%(\mathrm{n}=2,417)$ reported by Cravero et al., (2006). Fentanyl was used by the $\mathrm{RN}$ provider sample in combination with midazolam and pentobarbital in $8.39 \%(n=478)$ of cases (see Table 5). The frequency of medication combinations used by physicians and advanced practice providers were not reported by Cravero et al., (2006). Ketamine and propofol were administered in the RN sedation sample $1.49 \%(\mathrm{n}=188)$ and $0.18 \%(n=23)$ times respectively (see Figure 5$)$. In comparison, both ketamine and propofol were administered more often $13.6 \%(n=4,075)$ and $50.1 \%(n=15,059)$ of cases respectively in the sample reported by Cravero et al., (2006). The two most common medications administered by RNs were pentobarbital $48.16 \%(n=6,061)$ and chloral hydrate $43.29 \%(n=5,448)$ of the RN sample. More than one medication was delivered during $45.29 \%(n=5,699)$ of cases sedated by RNs (see Table 5).

\section{Discussion}

\section{Anesthesia Sedation Risk}

Patients sedated by RNs had similar ASA risk scores as Cravero's et al., (2006) large sample of patients sedated by physician and advanced practice providers. ASA risk score has been used by some organizations as a method to identify patients at a higher risk for sedation complications and to restrict RN sedation practice to patients with ASA scores of I or II (Lavoie et al., 2012). However, ASA III scores for this sample were similar to the physician provided sedation group reported by Cravero et al., (2006). This finding is important because it is unclear if RNs credentialed to provide sedation have completed training that prepares them to care for a patients with an ASA III risk score.

However, pediatric critical care experienced RNs do have the training and experience to care for patients who are severely ill. Pediatric Intensive Care Unit (PICU) RNs are required to have skills in drug administration, resuscitation, respiratory care, and the recognition and interpretation of various physiologic measures (Rosenberg \& Moss, 2004). Thus, the PICU RN may be better equipped with the skills and experience to deliver sedation to children up to and including ASA III than a general pediatric or radiology nurse who receives less training and demonstrates competency in sedation care but may not have other intensive care skills and experience to manage underlying coexisting conditions. 


\section{Adverse Events}

There are two types of adverse sedation events noted in this study: adverse events that may not require intervention such as desaturations that may be transient and not require treatment. The second type are unexpected adverse events that may require the RN to intervene, such as suctioning in order to manage patient secretions or prolonged sedation requiring the $\mathrm{RN}$ to extend the recovery period. It is notable that in this sample the most common unanticipated adverse event for the RN provider was prolonged sedation, which is often associated with the medications used for the procedure. However, it is not clear from these data how much longer than the usual recovery time was required when sedation was prolonged.

Prolonged sedation in children after a radiologic procedure has been previously reported by Malviya, Voepel-Lewis, Prochaska and Tait (2000) in their study of 376 children sedated by RNs for MRI and CT scan. They found that despite using the recommended sedation discharge criteria of returning to baseline vital signs, level of consciousness close to their baseline, and the ability to maintain a patent airway, $53 \%$ of children were asleep during their trip home from the hospital and 31\% were asleep for at least six hours after discharge from sedation for diagnostic MRI or CT scan (Malviya et al., 2000). Furthermore, motor imbalance was the most frequently reported side effect reported by parents, in one case persisting up to the day after sedation. In the study, 66\% of infants less than 12 months old had gross motor imbalance with the effect lasting for more than 6 hours (Malviya et al., 2000). Thus, it is possible that prolonged sedation may not be wholly evaluated at discharge and may require follow-up the day after sedation to obtain an accurate measure of the frequency of this adverse event. This may be accomplished by including a follow-up telephone call the day after the procedure. Some sedation services may already have this process in place as part of their quality improvement system. However, it is unknown if the questions being asked during follow-up telephone calls are eliciting information from parents regarding adverse events after discharge that do not require treatment, such as vomiting. Malviya and colleagues (2000) reported nausea and vomiting in 13\% of children after discharge from procedural sedation that resolved without treatment within six hours.

In this study sample, vomiting was the second most common sedation adverse event (see Figure 4) that may not require intervention by the RN, and was almost two times more frequent than in the physician sedation provider comparison group (Cravero et al., 2006). The increased frequency of vomiting with sedation by the $\mathrm{RN}$ alone group is likely related to the medication side effects or administration route used by RNs, such as the use of oral chloral hydrate that has a pungent odor and bitter taste (Chung, Hoffer, Connor, Zurakowski $\&$ Burrows, 2000). The most common medications administered either alone or in combination were pentobarbital, chloral hydrate and midazolam in this study sample (see Table 5).

\section{RN Sedation Practices}

Sedative Medication Use-Sedative medications are ordered by physicians or advance practice nurses. RNs often use preexisting protocols that delineate the drugs, routes, frequency, dosing, and indications for use by the sedating RN (Gozal, Drenger, Levin, 
Kadari \& Gozal, 2004; Lavoie et al., 2012). For example chloral hydrate can be given orally or rectally and is frequently used for MRI and CT scans because of its effectiveness and safety profile (Mason et al., 2004). However, it has a bitter taste that children may refuse to swallow or regurgitate, it has no reversal agent, can cause respiratory depression and prolonged sedation in some patients (Mason et al., 2004). In the Mason et al., (2004) study, chloral hydrate was the second most commonly administered medication by an RN. In the case of chloral hydrate, its use by RNs has persisted, despite evidence that other medications such as pentobarbital may be as effective and have fewer side effects. Pentobarbital was the most common sedative administered by RNs in this study sample, but it is not clear how or if RNs had a choice as to the sedative medication they administered.

Chloral hydrate alone or in combination with other medications such as diphenhydramine and meperidine has been compared with other sedatives such as oral midazolam and oral pentobarbital in small studies (Costa, Costa, Brasileiro, Bendo, Viegas \& Paiva; 2012; Mason et al., 2004). Most studies concerning chloral hydrate include samples of fewer than 1,000 patients with dosing variations (Costa et al., 2012). Chloral hydrate has been associated with adverse events ranging from deaths to vomiting (Costa et al., 2012). Adverse events were examined by Malviya et al., (2000) after discharge from procedural sedation. Chloral hydrate was the most common drug associated with motor imbalance and agitation; plus there were three reported episodes of prolonged sedation requiring an emergency department visit.

In this study RN use of fentanyl $n=1,158(9.2 \%)$, an opioid with analgesic properties, is of note because this study only included diagnostic procedures (MRI, CT scan and ultrasound) that are usually considered painless (see Figure 5). Fentanyl use by RNs was higher on a percentage basis when compared to other providers performing 12 possible categories of procedures $n=2,417$ (8\%) (Cravero et al., 2006). Conversely propofol was the most common medication delivered by other providers and was more common than RN use of propofol (Cravero et al., 2006). The medication administration patterns in this RN diagnostic radiology sample indicate that there is variation in $\mathrm{RN}$ sedation practices for diagnostic radiology when compared to practices by other sedation providers. Current training strategies emphasize a generalized approach at the organizational level rather than determining specific sedation practices by specialty and using that data to develop specialty specific training. However a generalized approach may fail to consider specialty specific sedation practices and the associated education needs of sedation providers. It is also unclear how prescribing decisions are made either by individual physicians or sedation committees that develop medication protocols. Additionally, many times, RNs administered combinations of medications that make each medication's effects less predictable.

The use of more than one medication is a risk factor for adverse events that has been identified in multiple studies (Coté et al., 2000; Lavoie et al., 2012). Numerous sedative and analgesic medications are available for use in procedural sedation and these can be administered in various combinations. However, it is difficult to study adverse events of combined sedative medications. One barrier is that a large sample of patients is necessary to collect enough data to determine which medication combinations provide the desired sedative effects with the fewest adverse events. 
Sedation Level-Due to the risks associated with sedation, the Joint Commission and the ASA have established sedation standards including credentialing requirements all sedation providers must meet (ASA, 2002; Joint Commission International, 2011). These sedation standards have focused on physician and advanced practice providers and offered limited guidance to RNs and their role in delivering sedation. Nursing professional standards and boards of nursing offer conflicting guidance on RN sedation practice. Crego (2013) reported on an in depth review of nursing sedation regulations in the United States. This descriptive study provides data on actual $\mathrm{RN}$ sedation practice that illustrates the disparity between RN sedation practice and $\mathrm{RN}$ sedation regulation.

Most sedation standards address RNs providing minimal to moderate sedation but in this sample RNs often planned and provided deep sedation to children undergoing diagnostic radiological procedures. For example, the ASA published a statement that only nonanesthesia physicians may be credentialed to provide deep sedation and may not delegate or supervise the administration or monitoring of deep sedation (ASA, 2012). Few boards of nursing and professional standards address deep sedation by RNs or have incorporated standards from organizations such as the ASA that restrict RNs from administering propofol and other anesthetic medications into their position statements. However, this study found that $13.69 \%$ of RNs in the sample provided deep sedation (see Table 5), demonstrating that RNs are already providing care that conflicts with recommendations by the ASA and exceeds the scope of practice set by some state boards of nursing (Davidson, Bloomberg, \& Burnell, 2007).

\section{Limitations}

The study sample was obtained using data collected by PSRC member institutions that self select and may represent organizations with highly organized sedation systems, with more experienced providers and sedation expertise than non-member institutions. Sedation outcomes in PSRC institutions may represent best practices rather than usual pediatric sedation outcomes. Although the PSRC includes data on provider types, characteristics of the provider are unknown, such as education level of RNs, clinical experience of the provider, or any specific sedation training requirements. Individual PSRC member institutions input data into the web based data collection tool, which may increase the chance of data entry errors due to the number of individuals entering data.

\section{Conclusions}

Sedation care is part of a complex system involving individual patient risk factors, sedation provider competency, medication choice and monitoring of the patient throughout and after the procedure is completed. This descriptive study indicates that there are several gaps in knowledge of sedation care by RNs in diagnostic radiology. Patients sedated by RNs had similar anesthesia risk scores to those sedated by physician and advanced practice providers, including some ASA III patients. However, most credentialing for RN sedation care providers assume the patient is at a low anesthesia risk. Sedation standards do not require $\mathrm{RN}$ sedation providers to have training and experience in caring for severely ill patients, instead they must be competent to rescue the patient from deep sedation or general 
anesthesia. However, the specific skills necessary for RNs to meet the rescue qualifications are not currently delineated and may also be dependent on the procedure length and location.

This study includes descriptions of current practices by RNs delivering and monitoring sedation. RNs in this study provided a deep level of sedation, despite restrictions on this practice by some professional organizations and regulatory bodies including state boards of nursing. The PSRC database excludes information on the location where sedation occurred so it is not possible to determine if RNs performing deep sedation were doing so outside their scope of practice, however this result may indicate that $\mathrm{RN}$ provided deep sedation may be occurring in some locations, despite the lack of uniform deep sedation standards. Adverse events in pediatric sedation may be closely related to the medications used, the type of procedure the child was sedated for, the monitoring completed throughout the procedure and follow-up procedures to detect adverse events after discharge. However, research on RN provided sedation has been done in single sites with small sample sizes that are insufficiently powered to provide adequate information on adverse events or to compare outcomes of RN sedation to other providers.

\section{Nursing Implications}

The results of this study provide evidence that the traditional assumptions regarding sedation practice by nurses may not accurately describe current practice and have several implications for nursing practice. The expansion of the role nurses have in sedation care requires a reevaluation of the monitoring practices and competencies nurses must have in order to safely provide sedation. In addition to the current emphasis on airway management, standardization of monitoring practices such as electrocardiogram and blood pressure monitoring should be changed to follow the current practice of other sedation providers such as physicians and advanced practice providers. The administration of medication combinations to induce deep sedation by RNs is another area that may not currently be included in sedation training and competency evaluation of sedation-credentialed nurses. The interaction and the potency of medications used in combination may be less predictable than when just one medication is administered to provide sedation. These differences may require additional knowledge and experience in order for sedative medication combinations to be administered and monitored safely by sedation nurses. Thus current sedation nurse credentialing methods may require that the specialty in which the nurse is practicing is considered in determining the competencies and skills necessary to provide sedation safely. Strategies to assure nurse sedation competency may include completion of a minimum number of deep sedations annually in order to be re-credentialed and the use of simulation to assess and maintain skills for management of sedation adverse events such as vomiting during procedures (Shavitz et al., 2007). In addition, knowledge testing of common sedative medications and combinations of medications used during procedures may need to be included in sedation nurses' training and credentialing process. A standardized set of questions to be used for follow-up telephone calls after sedation should be developed in order to determine if patients experienced adverse events after discharge from sedation services. Furthermore, the lack of nursing standards and regulation of deep sedation practice at the national and state level may require that an evaluation of the current practices of sedation by nurses is conducted in order to develop better nurse sedation practice standards 
across the United States. Further research on nurse sedation practice is necessary, as it is unknown if the practices described in this study are unique to sedation in pediatric patients in radiology or if similar practices are occurring in other pediatric specialty areas or in adults patients sedated by nurses.

\section{Future Research}

Although only cases in which sedation was monitored or delivered by RNs were included in this study, sedation involves other providers such as anesthesiologists who maintain responsibility for sedation cases even if they do not directly involve contact with the patient or involve more than one type of sedation provider during the procedure. Further research comparing sedation practices and outcomes by different types of providers is necessary to understand the significance of differences in patient risk factors, sedation practices, and the type of sedation provider on sedation outcomes. Differences in how sedation services are managed at the organizational level is also an area in which research is needed to determine how system level factors affect sedation outcomes. In addition, research is needed to determine outcomes of evidence based sedation education programs that considers the particulars of sedation by the type of procedure, location or specialty.

\section{Acknowledgments}

Financial Acknowledgement

This investigation was supported by the National Institutes of Health under Ruth L. Kirschstein National Research Service Award Grant Number: 1 F31 NR010426-01A1

\section{References}

American Society of Anesthesiologists. Advisory on Granting Privileges for Deep Sedation to NonAnesthesiologist Sedation Practitioners. 2010. Retrieved August 25, 2012, from http:// www.asahq.org/For-Members/Standards-Guidelines-and-Statements.aspx

American Society of Anesthesiologists. Statement on Granting Privileges to Nonanesthesiologists Physicians for Personally Administering or Supervising Deep Sedation. 2012. Retrieved February 1, 2013, from http://www.asahq.org/For-Members/Standards-Guidelines-and-Statements.aspx

American Society of Anesthesiologists Task Force on Anesthetic Care for Magnetic Resonance Imaging. Practice advisory on anesthetic care for magnetic resonance imaging: a report by the Society of Anesthesiologists Task Force on Anesthetic Care for Magnetic Resonance Imaging. Anesthesiology. 2009; 110(3):459-479.10.1097/ALN.0b013e31818f9206 [PubMed: 19237869]

American Society of Anesthesiologists Task Force on Sedation and Analgesia by NonAnesthesiologists. Practice guidelines for sedation and analgesia by non-anesthesiologists. Anesthesiology. 2002; 96(4):1004-1017. [PubMed: 11964611]

Beebe DS, Tran P, Bragg M, Stillman A, Truwitt C, Belani KG. Trained nurses can provide safe and effective sedation for MRI in pediatric patients. Canadian Journal of Anesthesia. 2000; 47(3):205210. [PubMed: 10730728]

Bluemke DA, Breiter SN. Sedation procedures in MR imaging: safety, effectiveness, and nursing effect on examinations. Radiology. 2000; 216(3):645-652. [PubMed: 10966690]

Chung T, Hoffer FA, Connor L, Zurakowski D, Burrows PE. The use of oral pentobarbital sodium (Nembutal) versus chloral hydrate in infants undergoing CT and MR imaging- a pilot study. Pediatric Radiology. 2000; 30(50):332-335. [PubMed: 10836598]

Costa LR, Costa PS, Brasileiro SV, Bendo CB, Viegas CM, Paiva SM. Post-discharge adverse events following pediatric sedation with high doses of oral medication. Journal of Pediatrics. 2012; 160(5): 807-813.10.1016/j.jpeds.2011.10.025 [PubMed: 22133425] 
Coté C, Wilson S. Guidelines for monitoring and management of pediatric patients during and after sedation for diagnostic and therapeutic procedures: an update. [Practice Guideline]. Pediatrics. 2006; 118(6):2587-2602.10.1542/peds.2006-2780 [PubMed: 17142550]

Couloures KG, Beach M, Cravero JP, Monroe KK, Hertzog JH. Impact of provider specialty on pediatric procedural sedation complication rates. Pediatrics. 2011; 127(5):e1154-1160.10.1542/ peds.2010-2960 [PubMed: 21518718]

Cravero JP, Beach ML, Blike GT, Gallagher SM, Hertzog JH. The incidence and nature of adverse events during pediatric sedation/anesthesia with propofol for procedures outside the operating room: a report from the Pediatric Sedation Research Consortium. Anesthesia and Analgesia. 2009; 108(3):795-804.10.1213/ane.0b013e31818fc334 [PubMed: 19224786]

Cravero JP, Blike GT, Beach M, Gallagher SM, Hertzog JH, Havidich JE, Gelman B. Incidence and nature of adverse events during pediatric sedation/anesthesia for procedures outside the operating room: report from the Pediatric Sedation Research Consortium. Pediatrics. 2006; 118(3):10871096.10.1542/peds.2006-0313 [PubMed: 16951002]

Crego N. Procedural Sedation in the United States: A Review of Nursing Regulation. 2013 Unpublished manuscript.

Davidson JE, Bloomberg D, Burnell L. Scope creep: when nursing practice moves beyond traditional boundaries: an evidence-based example using procedural sedation. Critical Care Nursing Quarterly. 2007; 30(3):219-232.10.1097/01.CNQ.0000278922.21821.52 [PubMed: 17579305]

Godwin SA, Caro DA, Wolf SJ, Jagoda AS, Charles R, Marett BE, Moore J. Clinical policy: procedural sedation and analgesia in the emergency department. Annals of Emergency Medicine. 2005; 45(2):177-196.10.1016/j.annemergmed.2004.11.002 [PubMed: 15671976]

Gozal D, Drenger B, Levin PD, Kadari A, Gozal Y. A pediatric sedation/anesthesia program with dedicated care by anesthesiologists and nurses for procedures outside the operating room. Journal of Pediatrics. 2004; 145(1):47-52.10.1016/j.jpeds.2004.01.044 [PubMed: 15238906]

Gozal D, Gozal Y. Pediatric sedation/anesthesia outside the operating room. Current Opinion in Anaesthesiology. 2008; 21(4):494-498.10.1097/ACO.0b013e3283079b6c [PubMed: 18660660]

Havidich JE, Cravero JP. The current status of procedural sedation for pediatric patients in out-ofoperating room locations. Current Opinion in Anaesthesiology. 2012; 25(4):453-460.10.1097/ ACO.0b013e32835562d8 [PubMed: 22732423]

Joint Commission International. Comprehensive Accreditation Manual for Hospitals. Oak Brook: Joint Commission Resources; 2011.

Krauss B, Green SM. Training and credentialing in procedural sedation and analgesia in children: lessons from the United States model. Paediatric Anaesthesia. 2008; 18(1):30-35.10.1111/j. 1460-9592.2007.02406.x [PubMed: 18095963]

Langhan ML, Mallory M, Hertzog J, Lowrie L, Cravero J. Physiologic monitoring practices during pediatric procedural sedation: a report from the Pediatric Sedation Research Consortium. Archives of Pediatric and Adolescent Medicine. 2012; 166(11):990-998.10.1001/archpediatrics.2012.1023

Lavoie L, Vezina C, Paul-Savoie E, Cyr C, Lafrenaye S. Procedural pediatric sedation by nurses: available, competent, and safe. International Journal of Pediatrics. 2012; 2012:15.10.1155/2012/820209

Malviya S, Voepel-Lewis T, Prochaska G, Tait AR. Prolonged recovery and delayed side effects of sedation for diagnostic imaging studies in children. Pediatrics. 2000; 105(3):E42. [PubMed: 10699144]

Mason KP, Sanborn P, Zurakowski D, Karian VE, Connor L, Fontaine PJ, Burrows PE. Superiority of pentobarbital versus chloral hydrate for sedation in infants during imaging. Radiology. 2004; 230(2):537-542.10.1148/radiol.2302030107 [PubMed: 14699175]

Rosenberg DI, Moss MM. Guidelines and levels of care for pediatric intensive care units. Pediatrics. 2004; 114(4):1114-1125.10.1542/peds.2004-1599 [PubMed: 15466118]

Shah A, Mosdossy G, McLeod S, Lehnhardt K, Peddle M, Rieder M. A blinded, randomized controlled trial to evaluate ketamine/propofol versus ketamine alone for procedural sedation in children. Annals of Emergency Medicine. 2011; 57(5):425-433. e422.10.1016/j.annemergmed. 2010.08.032 [PubMed: 20947210] 
Shavit I, Keidan I, Hoffmann Y, Mishuk L, Rubin O, Ziv A, Steiner IP. Enhancing patient safety during pediatric sedation: the impact of simulation-based training of nonanesthesiologists. Archives of Pediatric and Adolescent Medicine. 2007; 161(8):740-743.10.1001/archpedi. 161.8.740

Srinivasan M, Turmelle M, Depalma LM, Mao J, Carlson DW. Procedural sedation for diagnostic imaging in children by pediatric hospitalists using propofol: analysis of the nature, frequency, and predictors of adverse events and interventions. Journal of Pediatrics. 2012; 160(5):801-806. e801.10.1016/j.jpeds.2011.11.003 [PubMed: 22177990]

Sterni L, Beck S, Cole J, Carlson D, Turmelle M. A model for pediatric sedation centers using pharmacologic sedation for successful completion of radiologic and procedural studies. Journal of Radiology Nursing. 2008; 27(2):46-60.10.1016/j.jradnu.2008.01.004

Sury MR, Hatch DJ, Deeley T, Dicks-Mireaux C, Chong WK. Development of a nurse-led sedation service for paediatric magnetic resonance imaging. Lancet. 1999; 353(9165):1667-1671. [PubMed: 10335787]

Woodthorpe C, Trigg A, Gurney A, Sury M. Nurse led sedation for paediatric MRI: progress and issues. Paediatric Nursing. 2007; 19(2):14-18. [PubMed: 17425124] 


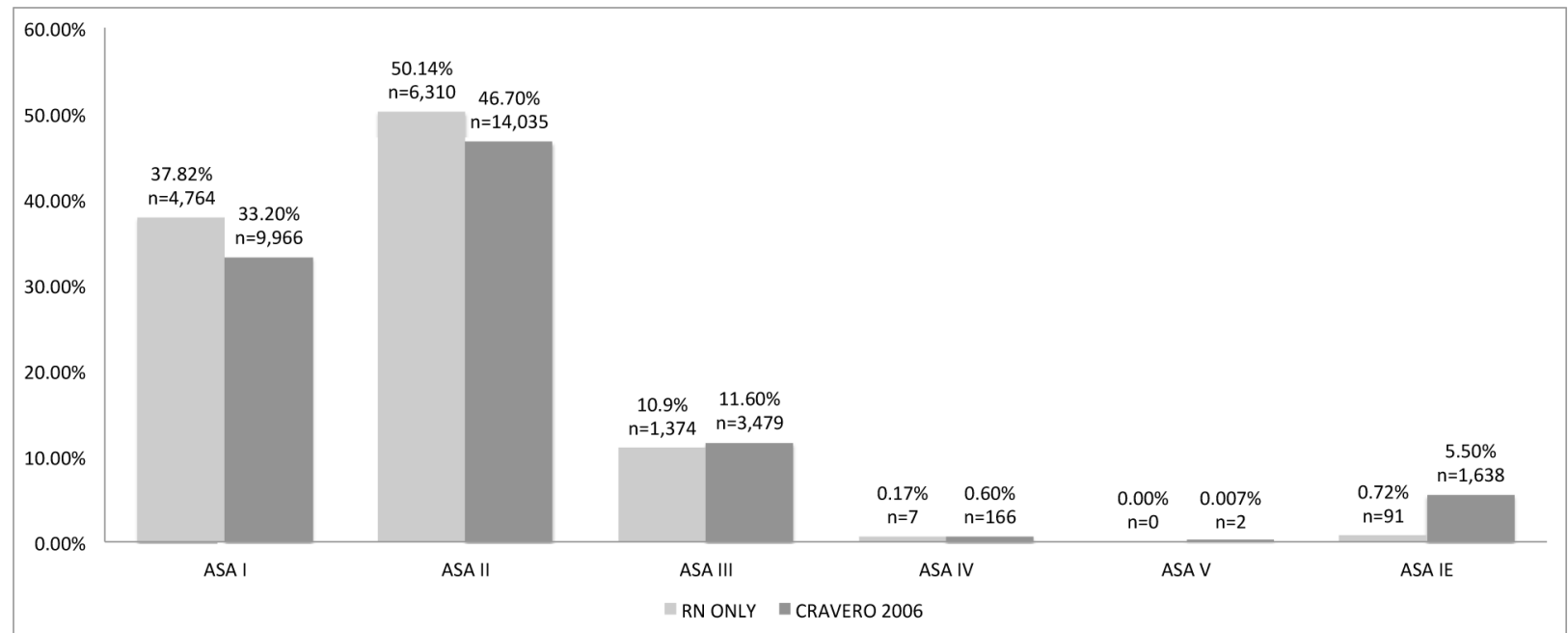

Figure 1.

Comparison between nurse and other sedation providers ASA scores ${ }^{1}$ ASA scores for sedation delivered and monitored by nurses were obtained from a sample of 12,584 cases. Comparison data were obtained from the Pediatric Sedation Research Consortium sample of physicians and advanced practice nurses on 30,037 sedation cases reported by Cravero et al., (2006). 


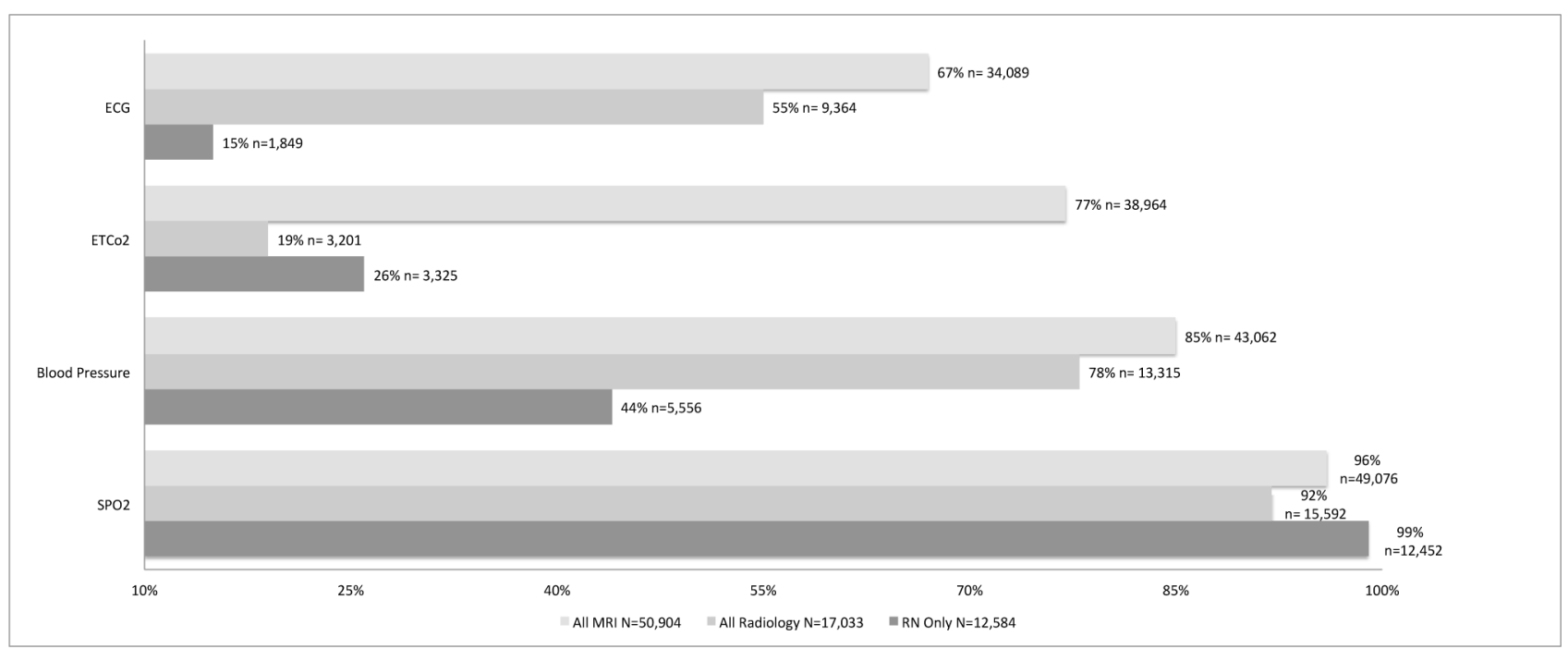

Figure 2.

Comparison of monitoring equipment use for sedation cases by physician and nurse providers in diagnostic radiology

${ }^{1}$ Monitoring data for nurse delivered sedation includes MRI, CT scan and ultrasound in a sample of 12,584 cases. Comparison physician monitoring data was obtained from the Pediatric Sedation Research Consortium sample of 50,904 sedation cases in MRI and other radiology procedures reported by Langhan et al., (2012). 


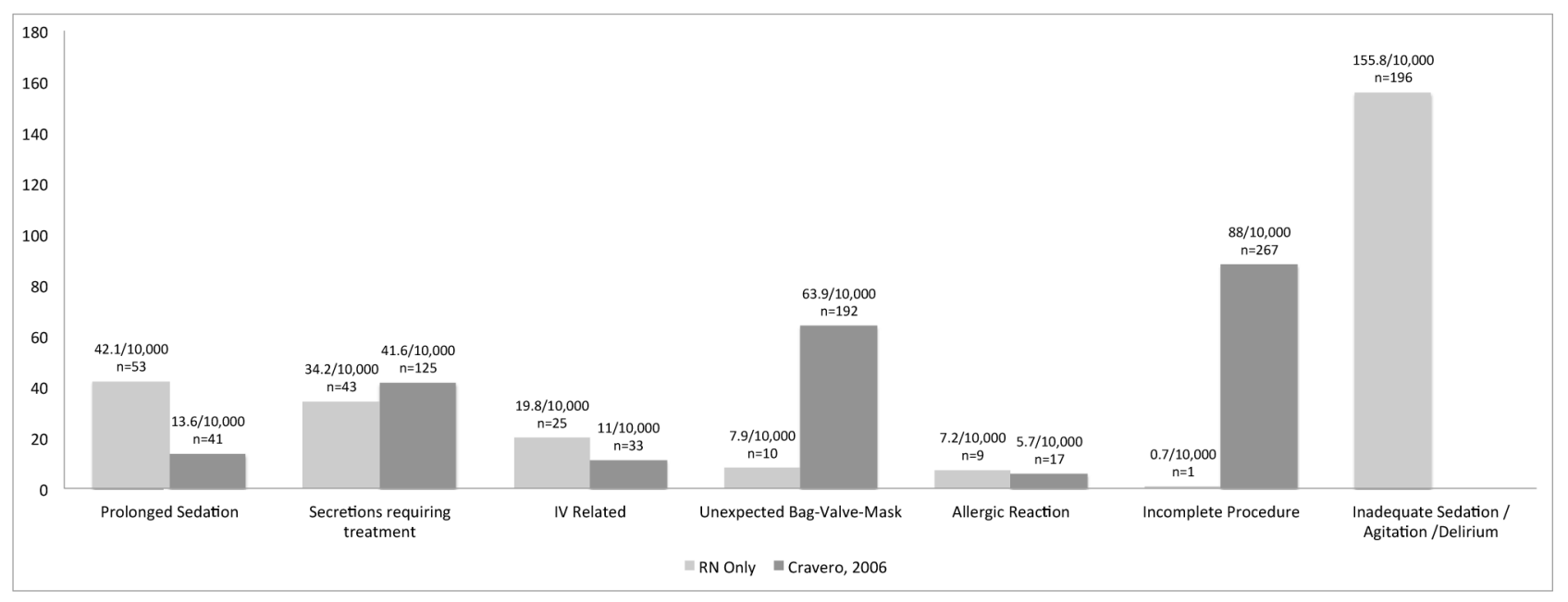

Figure 3.

Comparison of unexpected adverse events per 10,000 between nurse and other sedation providers

${ }^{1}$ Unexpected adverse events for sedation delivered and monitored by nurses were obtained from a sample of 12,584 cases. Comparison data of unexpected events was obtained from the Pediatric Sedation Research Consortium sample of physicians and advanced practice nurses on 30,037 sedation cases reported by Cravero et al.,(2006). RN only sample of inadequate sedation/agitation/delirium was not reported by Cravero et al., (2006). 


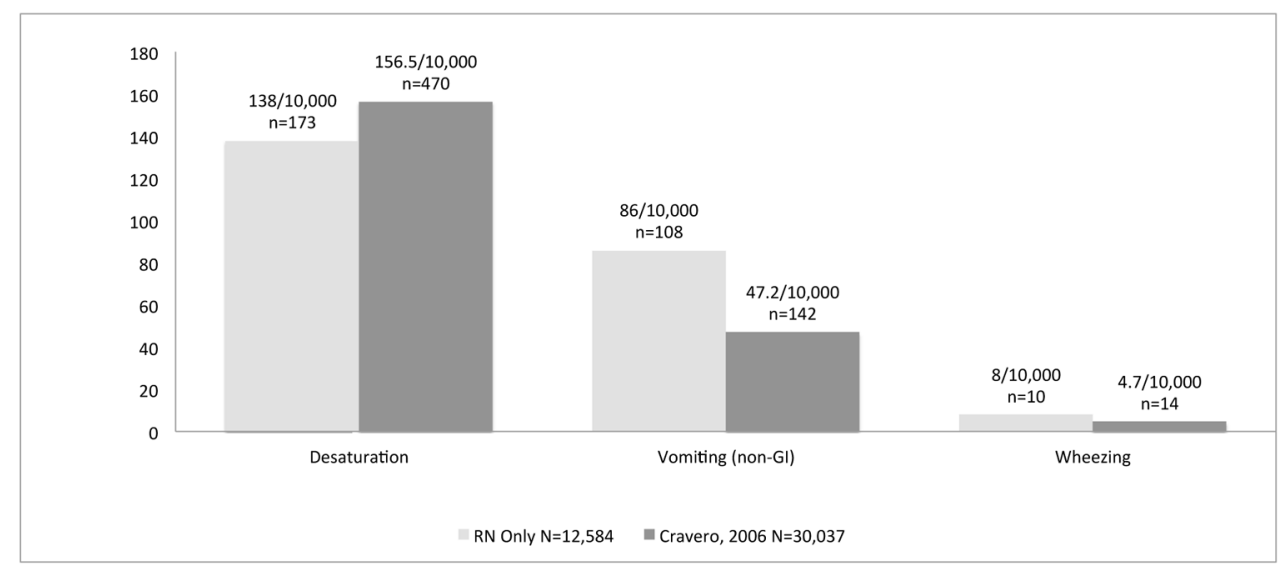

Figure 4.

Frequency of nurse and other sedation provider adverse events that may not require interventions

${ }^{1}$ Adverse events for sedation delivered and monitored by nurses were obtained from a sample of 12,584 cases. Comparison data of adverse events was obtained from the Pediatric Sedation Research Consortium sample of physicians and advanced practice nurses on 30,037 sedation cases reported by Cravero et al., (2006). 


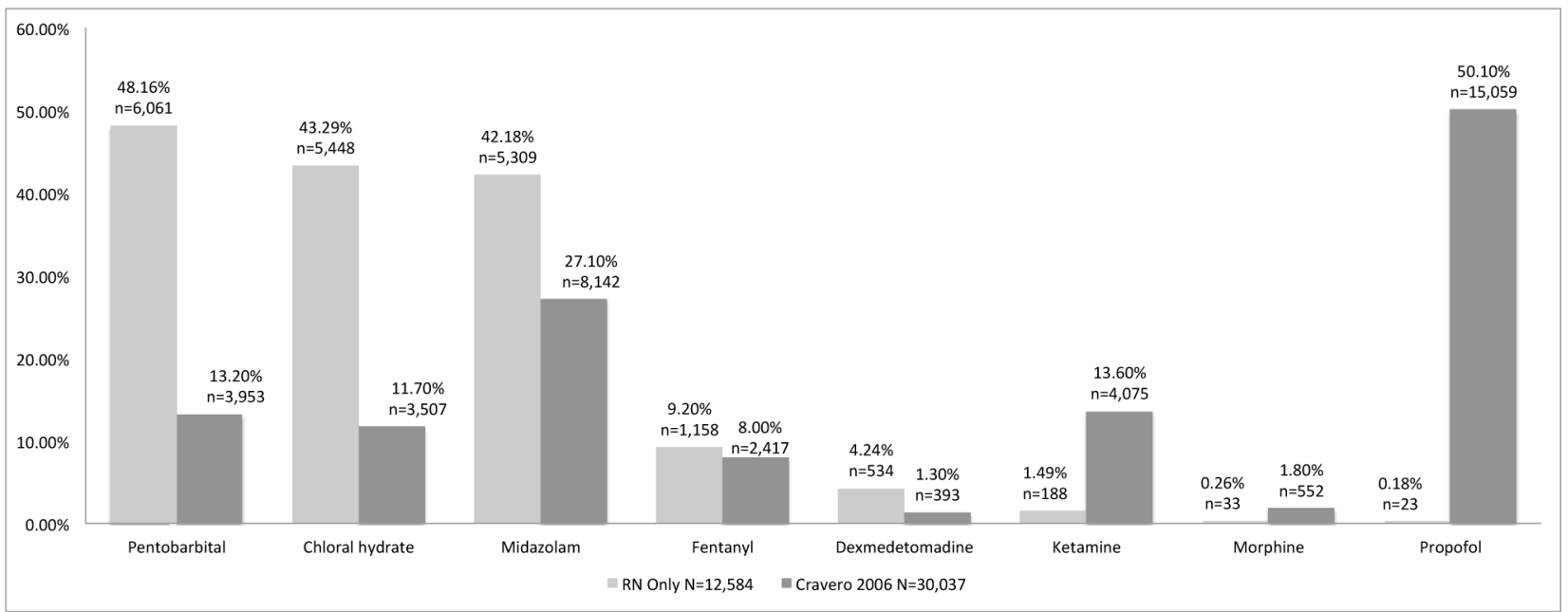

Figure 5.

Medication use by nurses and other sedation providers.

${ }^{1}$ Medication use for sedation delivered and monitored by nurses was obtained from a sample of 12,584 cases. Comparison data was obtained from the Pediatric Sedation Research Consortium, a sample of physicians and advanced practice nurses on 30,037 sedation cases reported by Cravero et. al, (2006). 
Table 1

American Society of Anesthesiologists Sedation Level Definitions

Sedation Level Definition 1

Minimal Sedation (anxiolysis) Level

A drug induced state in which patients respond normally to verbal commands. Although cognitive function and coordination may be impaired ventilation and cardiovascular function are usually maintained.

Moderate Sedation Level

A drug-induced depression of consciousness in which patients respond purposefully to verbal commands alone or when accompanied by light tactile stimulation. The patient maintains their airway without intervention and spontaneous ventilation is adequate. Cardiovascular function usually is maintained.

Deep Sedation Level

A drug-induced depression of consciousness in which patients cannot be aroused easily; respond purposefully following repeated or painful stimulation. The patient maintains independent ventilatory function that may be impaired. Patients may require assistance to maintain airway patency, spontaneous ventilation may be inadequate. Cardiovascular function usually is maintained.

\section{General Anesthesia Level}

A drug-induced loss of consciousness in which patients are not arousable, even by painful stimulation. Ability to independently maintain ventilatory function is often impaired. Patients often require assistance in maintaining a patent airway, positive pressure ventilation may be required due to depressed spontaneous ventilation or drug-induced depression of neuromuscular function. Cardiovascular function may be impaired.

${ }^{1}$ Adapted from American Society of Anesthesiologist Task Force on Sedation and Analgesia by Non-Anesthesiologists. (2002). Practice guidelines for sedation and analgesia by non-anesthesiologists. Anesthesiology, 96(4), 1004-1017. 


\section{Table 2}

\section{American Society of Anesthesiologists physical status classification}

\section{Physical Status Classification}

Class I - Normally healthy patient

Class II - Patient with mild systemic disease

Class III - Patient with severe systemic disease

Class IV - Patient with a severe systemic disease that is a constant threat to life

Class V - A moribund patient who is not expected to survive without the operation

Class IE, IIE, IIIE, IVE - An "E" Suffix can be added to each ASA class to indicate an emergency surgery/procedure

${ }^{1}$ Adapted from American Society of Anesthesiologist Task Force on Sedation and Analgesia by Non-Anesthesiologists. (2002). Practice guidelines for sedation and analgesia by non-anesthesiologists. Anesthesiology, 96(4), 1004-1017. 
Table 3

Sample Inclusion criteria, number of excluded case and sample size

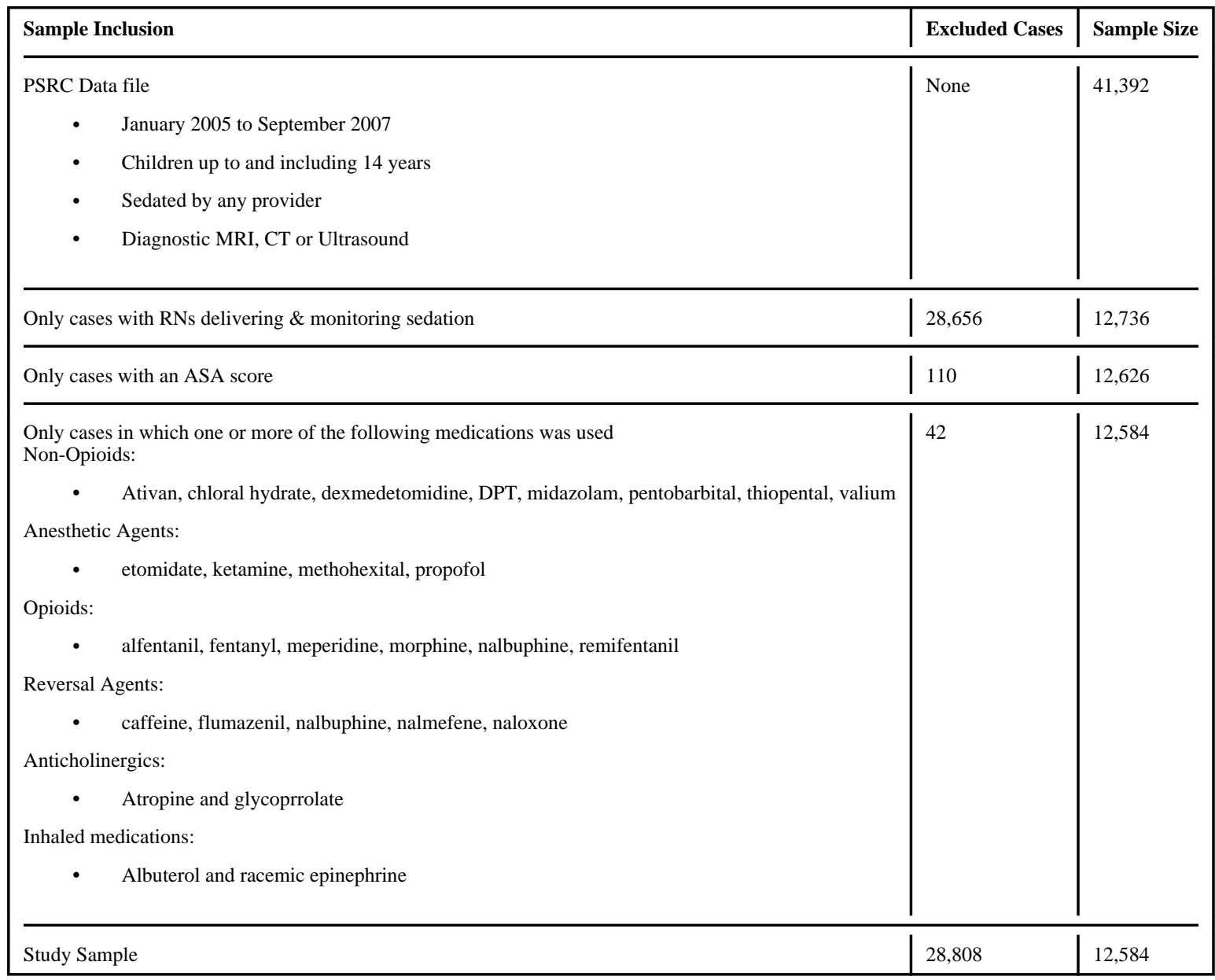


Table 4

Sample characteristics of pediatric patient cases that were monitored and received medications for sedation by an $\mathrm{RN}$ in diagnostic radiology

\begin{tabular}{|c|c|c|c|}
\hline \multirow{2}{*}{$\begin{array}{l}\text { Variable } \\
\text { Weight in kilograms }\end{array}$} & \multirow{2}{*}{$\frac{N}{}$} & \multicolumn{2}{|c|}{ Descriptive Statistics } \\
\hline & & $\begin{array}{c}\text { Mean } \\
\text { Standard Deviation } \\
\text { Range }\end{array}$ & $\begin{array}{c}15.08 \\
9.5 \\
0.5-236\end{array}$ \\
\hline \multirow[t]{2}{*}{ Age in months } & 12,584 & $\begin{array}{c}\text { Mean } \\
\text { Standard Deviation } \\
\text { Range }\end{array}$ & $\begin{array}{c}34.9 \\
32.2 \\
0-168\end{array}$ \\
\hline & $N$ & \multicolumn{2}{|l|}{ Percent } \\
\hline \multicolumn{4}{|l|}{ Age in Categories } \\
\hline Infant (0-12 month) & 3,688 & 29.31 & \\
\hline Toddler (13 - 24 month) & 2,565 & 20.38 & \\
\hline Early Childhood ( 25 months -5 years) & 4,423 & 35.15 & \\
\hline Middle Childhood (6 - 11 years) & 1,693 & 13.45 & \\
\hline Teen $(12-14$ years $)$ & 215 & 1.71 & \\
\hline \multicolumn{4}{|l|}{ Sex } \\
\hline Male & 6,979 & 55.46 & \\
\hline Female & 5,591 & 44.43 & \\
\hline Missing & 14 & 0.11 & \\
\hline \multicolumn{4}{|l|}{${ }^{1}$ Categories of Primary Diagnosis } \\
\hline Cardiovascular & 168 & 1.34 & \\
\hline Craniofacial Abnormalities & 467 & 3.71 & \\
\hline Gastrointestinal & 213 & 1.69 & \\
\hline Metabolic/Genetics (includes obesity) & 359 & 2.85 & \\
\hline Neurological & 7,381 & 58.65 & \\
\hline Prematurity Related & 56 & 0.45 & \\
\hline Respiratory - Lower airway & 128 & 1.02 & \\
\hline Respiratory- Upper airway & 140 & 1.11 & \\
\hline \multicolumn{4}{|l|}{2 Categories of Coexisting Diagnosis } \\
\hline Cardiovascular & 519 & 4.12 & \\
\hline Craniofacial Abnormalities & 80 & 0.64 & \\
\hline Gastrointestinal & 1,004 & 7.98 & \\
\hline Metabolic/Genetics (includes obesity) & 425 & 3.38 & \\
\hline Neurological & 1,851 & 14.71 & \\
\hline Prematurity Related & 335 & 2.66 & \\
\hline Respiratory - Lower airway & 1,196 & 9.50 & \\
\hline
\end{tabular}




\begin{tabular}{|l|c|c|}
\hline Variable & $N$ & Descriptive Statistics \\
\hline \multicolumn{1}{|c|}{ Respiratory- Upper airway } & 2,491 & 19.79 \\
\hline Number of Coexisting Conditions/Case & & \\
None & 7,086 & 56.39 \\
One & 3,800 & 30.20 \\
Greater than 1 coexisting condition & 1,688 & 13.43 \\
\hline
\end{tabular}

${ }^{1}$ Selected primary diagnosis categories out of 24 possible choices

${ }^{2}$ Selected coexisting diagnosis categories out of 24 possible choices 
Table 5

Description of providers responsible for sedation and characteristics of diagnostic radiology procedures in a sample of cases of sedation monitored and delivered by an $\mathrm{RN}$

\begin{tabular}{|c|c|c|}
\hline Variable & $N$ & Percent \\
\hline \multicolumn{3}{|l|}{ Sedation Provider Information } \\
\hline \multicolumn{3}{|l|}{1 Provider Ultimately Responsible for Sedation } \\
\hline Anesthesiologists & 4,339 & 34.54 \\
\hline Pediatrician & 2,909 & 23.15 \\
\hline APRN/PNP/PA & 2,299 & 18.3 \\
\hline Intensivist & 1,404 & 11.17 \\
\hline Emergency Medicine Physician & 1,264 & 10.06 \\
\hline Radiologist & 190 & 1.51 \\
\hline Other & 108 & 0.86 \\
\hline Fellowship Level & 28 & 0.22 \\
\hline $\mathrm{RN}$ & 18 & 0.14 \\
\hline House Staff & 3 & 0.02 \\
\hline Missing Data & 2 & 0.02 \\
\hline CRNA & 0 & 0 \\
\hline RN alone Monitoring and delivering sedation & 12,584 & 100 \\
\hline \multicolumn{3}{|l|}{ Diagnostic Radiology Procedure Characteristics } \\
\hline \multicolumn{3}{|l|}{2 Diagnostic Radiologic Procedures } \\
\hline MRI Scan & 8,802 & 70.06 \\
\hline CT Scan & 3,861 & 30.73 \\
\hline Ultrasound & 61 & 0.49 \\
\hline \multicolumn{3}{|l|}{ Depth of Sedation Planned } \\
\hline Minimal & 374 & 2.97 \\
\hline Moderate & 10,462 & 83.12 \\
\hline Deep & 1,741 & 13.84 \\
\hline General Anesthesia & 7 & 0.06 \\
\hline \multicolumn{3}{|l|}{ Depth of Sedation Achieved } \\
\hline Minimal & 433 & 3.44 \\
\hline Moderate & 10,422 & 82.82 \\
\hline Deep & 1,723 & 13.69 \\
\hline General Anesthesia & 6 & 0.05 \\
\hline 3 Total Adverse Events & 727 & 5.78 \\
\hline \multicolumn{3}{|l|}{${ }^{4}$ Adverse Events by Category } \\
\hline Respiratory Complications & 297 & 2.36 \\
\hline
\end{tabular}




\begin{tabular}{|l|c|c|}
\hline Variable & $N$ & Percent \\
\hline Neurological Complications & 262 & 2.08 \\
Other Complications & 221 & 1.76 \\
Cardiovascular Complications & 34 & 0.27 \\
Emergent Complications & 19 & 0.15 \\
\hline Non-Sedative Medication Used & & \\
Glycopyrrolate & 279 & 2.22 \\
Albuterol & 146 & 1.17 \\
Atropine & 109 & 0.87 \\
Flumazenil & 1 & 0.008 \\
Naloxone & 1 & 0.008 \\
\hline Number of Medications per Case & & \\
1 medication & 6,885 & 54.71 \\
2 medications & 5,209 & 41.39 \\
3 medications & 484 & 3.85 \\
4 medications & 6 & 0.05 \\
\hline Most Common Combination of Medications & 3,572 & 62.68 \\
Midazolam and pentobarbital & 716 & 12.56 \\
Pentobarbital and fentanyl & 478 & 8.39 \\
Dexmedetomidine and midazolam & 401 & 7.04 \\
Midazolam, pentobarbital, fentanyl & 232 & 4.07 \\
Chloral hydrate and midazolam & 149 & 2.61 \\
Midazolam and ketamine & & \\
& & \\
\hline
\end{tabular}

${ }^{I}$ The provider responsible reflects the provider responsible for the sedation case but does not mean the provider had contact with the patient ${ }^{2}$ Cases may have more than one diagnostic radiology procedure

${ }^{3}$ Adverse Events are defined as any case with reported complication

${ }^{4}$ Cases may contain more than one category of adverse events 